\title{
Gradient Digitization Error Artifact
}

National Cancer Institute

\section{Source}

National Cancer Institute. Gradient Digitization Error Artifact. NCI Thesaurus. Code C86991.

An artifact that results from a gradient which is not constant with respect to the gradient direction. 